\title{
Gravitational Instability of a Kink
}

\author{
W. Barreto ${ }^{1,2}$, R. Gómez ${ }^{1}$, L. Lehner ${ }^{1}$ and J. Winicour ${ }^{1}$ \\ ${ }^{1}$ Department of Physics and Astronomy, University of Pittsburgh, Pittsburgh, PA 15260 \\ ${ }^{2}$ Laboratorio de Física Teórica, Departamento de Física, Escuela de Ciencias, Núcleo de Sucre \\ Universidad de Oriente, Cumaná, Venezuela
}

\begin{abstract}
We study the equilibria of a self-gravitating scalar field in the region outside a reflecting barrier. By introducing a potential difference between the barrier and infinity, we create a kink which cannot decay to a zero energy state. In the realm of small amplitude, the kink decays to a known static solution of the Einstein-Klein-Gordon equation. However, for larger kinks the static equilibria are degenerate, forming a system with two energy levels. The upper level is unstable and, under small perturbations, decays to the lower energy stable equilibrium. Under large perturbations, the unstable upper level undergoes collapse to a black hole. The equilibrium of the system provides a remarkably simple and beautiful illustration of a turning point instability.
\end{abstract}

04.40.-b,04.25.Dm,04.30.Db 


\section{INTRODUCTION}

We describe here a simple model of a system exhibiting a gravitationally induced transition from an unstable static equilibrium to a lower energy stable equilibrium (for small perturbations) or to a black hole (for large perturbations). The system is the general relativistic version of a spherically symmetric scalar wave $\Phi$ reflecting off an inner mirror of radius $R$. By holding the mirror at a fixed potential with respect to infinity, we create kink boundary conditions which do not allow decay of the field to a zero energy state. In the nongravitating case, any initial configuration would decay to the static solution of the wave equation, $\Phi=A R / r$, where $A$ is the potential of the mirror; and this static solution is stable under perturbations. In the self-gravitating case we will show there is a critical value of kink potential $A_{c}$ about which the system displays a classic example of a turning point instability $[1,2]$, responsible for the above phenomena. Above this critical potential, there are no equilibria and all configurations collapse to a black hole. The behavior of this system illustrates the remarkable richness of the physical properties that result from the mixture of long range gravitational forces and nonlinearity.

Our theoretical model is the null-timelike initial value problem for a spherically symmetric space-time satisfying the coupled Einstein-Klein-Gordon equations for a massless scalar field. We pose this mixed boundary value problem in the region of space-time outside a timelike inner boundary and to the future of an initial null hypersurface. The mathematical details of this problem have been described before in an investigation of the decay of scalar wave tails [3]. In that case, the scalar field was set to zero on the inner boundary, so that the boundary acted as a perfectly reflecting mirror. Here, by holding the inner boundary at a fixed potential, its role as a mirror is unchanged due to the local gauge invariance of the system with respect to the transformation $\Phi \rightarrow \Phi+$ constant. However, the potential difference with respect to infinity introduces nontrivial global effects.

We set the geometry of the mirror to correspond to a surface of radius $R$ enclosing a flat region of spacetime. The evolution of the system then depends uniquely upon the characteristic data for the scalar field on the initial null hypersurface, which extends from the mirror to future null infinity $\mathcal{I}^{+}$.

We describe this system in terms of a null coordinate system with the spherically symmetric line element

$$
d s^{2}=e^{2 \beta} d u\left(\frac{V}{r} d u+2 d r\right)-r^{2}\left(d \theta^{2}+\sin ^{2} \theta d \phi^{2}\right) .
$$

In these coordinates, the Einstein-Klein-Gordon equations reduce to $[4,5]$

$$
\begin{gathered}
\beta_{, r}=2 \pi r\left(\Phi_{, r}\right)^{2} \\
V_{, r}=e^{2 \beta}
\end{gathered}
$$

and the scalar wave equation $\square \Phi=0$, which takes the form

$$
2(r \Phi)_{, u r}=r^{-1}\left(r V \Phi_{, r}\right)_{, r}
$$


The initial null data necessary for evolution consists of $\Phi\left(u_{0}, r\right), r \geq R$, at initial time $u_{0}$. (We take $u_{0}=0$ ). At the mirror, we set $\Phi(u, R)=A=$ constant, with the gauge condition that $\Phi(u, \infty)=0$. We adopt the coordinate condition $\beta(u, R)=0$. The condition that the metric match continuously to a flat interior for $r<R$ requires $V(u, R)=R$. (The normal derivatives of $\Phi$ and the metric do not match continuously across $r=R$ in accord with the sheet stresses associated with the mirror).

With these conditions the scalar field and metric components have a unique future evolution. The resulting metric does not have an asymptotic Minkowski form at $\mathcal{I}^{+}$. This is characterized by the quantity $H(u)=\beta(u, \infty)$ which relates Bondi time $t$ at $\mathcal{I}^{+}$to the proper time $\tau=u$ at the reflecting boundary according to $d t / d \tau=e^{2 H}$. Bondi time is the physically relevant time for distant observers. The Bondi mass of the system can be expressed in either an asymptotic or integral form [6]:

$$
\begin{aligned}
M(u) & =\left.\frac{1}{2} e^{-2 H} r^{2}\left(\frac{V}{r}\right)_{, r}\right|_{r=\infty} \\
& =2 \pi \int_{R}^{\infty} e^{2(\beta-H)} r^{2}\left(\Phi_{, r}\right)^{2} d r .
\end{aligned}
$$

(Note that the mass content of a null hypersurface only depends upon field values in the hypersurface and is independent of their time derivative).

In the flat space limit, (2) and (3) imply $\beta=0$ and $V=r$ and (4) reduces to the wave equation in null-radial coordinates:

$$
\square^{(2)} g:=2 g_{, u r}-g_{, r r}=0,
$$

where $g=r \Phi$. The general solution satisfying the boundary condition for a kink potential $A$ is

$$
g(u, r)=A R+f(u / 2+r)-f(u / 2+R) .
$$

The first term describes a static monopole; the second, an incoming wave; and the third, its reflection off the mirror. At late retarded time, the solution asymptotically approaches the static equilibrium state $\Phi \rightarrow A R / r$. This state is also the solution of the variational problem for the energy, $\delta M=0$ subject to the constraint that $\delta \Phi$ vanishes at the mirror and at infinity. In this flat space example,

$$
M=2 \pi \int_{R}^{\infty}\left(\Phi_{, r}\right)^{2} r^{2} d r
$$

and its variation is given by

$$
\delta M=-4 \pi \int_{R}^{\infty}\left[\Phi_{, r} r^{2}\right]_{, r} \delta \Phi d r .
$$

In the flat space case, the static solution $\Phi=A / r$ is the unique extremum satisfying the boundary conditions (which rule out the trivial solution $\Phi=$ constant). Furthermore, the second variation of the mass about this state is positive, so that it represents a stable equilibrium, which the system approaches after radiating its excess energy to infinity. 
In Sec.II, we show that for small kink potentials these features extend to the curved space, self-gravitating case. But, above the critical kink potential, equilibria do not exist. We show that this critical potential marks a turning point instability about which the equilibrium configurations bifurcate into stable and unstable form. In Sec.III, we present numerical simulations of the evolution of stable and unstable kinks.

\section{ENERGETICS OF SELF-GRAVITATING KINKS}

At the initial retarded time, the null data $\Phi(0, r)$ and the constrained kink potential $A=\Phi(u, R)$ uniquely determine the future evolution of the system. In order to discuss the energetics of these states it is convenient to describe the corresponding configuration space in terms of $\Phi(V)=\Phi(0, r(V))$, where $V$ is considered the independent variable. Here $V$, as determined by (2) and (3), is the affine parameter along the outgoing null cone. The affine freedom is fixed by the boundary conditions $V(R)=r(R)=R$ and $r^{\prime}(R)=1$, where we denote $\partial_{V} f=f^{\prime}$. Then (2) and (3) can be reexpressed as $r=f$, where $f$ satisfies

$$
f^{\prime \prime}=-4 \pi\left(\Phi^{\prime}\right)^{2} f
$$

which determines $r(V)$ uniquely in terms of the initial data. The mass of of the state $\Phi(V)$ can be obtained by inserting the solution $r(V)$ of (10) into the first version of (5), which gives

$$
M=\left.\frac{1}{2}\left(r-V r^{\prime}\right)\right|_{\infty},
$$

when reexpressed with $V$ as the independent variable.

\section{A. Static equilibria}

We now show that the asymptotically flat static solution $\Psi$ of the Einstein-Klein-Gordon system is an extremum of the energy, subject to a fixed kink potential. This solution [7], which is the analog of the $1 / r$ solution in a Minkowski background, can be obtained in null coordinates by setting $\Phi_{, u}=0$ in the wave equation (4). This gives

$$
r V \Psi_{, r}=\text { const }
$$

whose solution, after using (2) and (3) to eliminate the $r$-dependence, is

$$
\Psi(V)=\frac{1}{4 \sqrt{\pi} \cosh \alpha} \ln \left[\frac{V+R\left(e^{2 \alpha}-1\right)}{V+R\left(e^{-2 \alpha}-1\right)}\right]
$$

with $r(V)=r_{\Psi}$ given by

$$
r_{\Psi}^{2}=e^{-4 \alpha \tanh \alpha}\left[V+R\left(e^{-2 \alpha}-1\right)\right]^{1-\tanh \alpha}\left[V+R\left(e^{2 \alpha}-1\right)\right]^{1+\tanh \alpha} .
$$

Here the integration constant $\alpha$ determines the kink potential, 


$$
A_{\Psi}(\alpha)=\frac{\alpha}{\sqrt{\pi} \cosh \alpha}
$$

The spacetime has a naked singularity when analytically extended to $r=0$ [7]. The Bondi mass of this solution is

$$
M_{\Psi}(\alpha)=2 R \sinh ^{2} \alpha e^{-2 \alpha \tanh \alpha} .
$$

In order to investigate the behavior of the Bondi mass with respect to variations about $\Psi$, we consider the solutions of the second order differential equation (10) for an arbitrary configuration $\Phi$. The solution $r$ is uniquely determined by the boundary conditions $r(R)=R$ and $r^{\prime}(R)=1$ and has asymptotic behavior $r \sim e^{-2 H} V+2 M+O(1 / V)$ as $V \rightarrow \infty$. We define a second independent solution $\tau$ by requiring asymptotic behavior $\tau \sim V+O(1 / V)$. For a general configuration $\Phi$, this normalizes the Wronskian of these solutions to the Bondi mass,

$$
2 M=r \tau^{\prime}-\tau r^{\prime}
$$

Now, for a fixed kink potential, consider the $\epsilon$-dependent family of perturbed configurations $\Phi=\Psi+\epsilon \phi$, subject to the boundary condition $\phi(R)=\phi(\infty)=0$. For $\epsilon=0$, we have $r=r_{\Psi}$ and $\tau=\tau_{\Psi}$, with $r_{\Psi}$ given by (14) and

$$
\tau_{\Psi}^{2}=\left[V+R\left(e^{-2 \alpha}-1\right)\right]^{1+\tanh \alpha}\left[V+R\left(e^{2 \alpha}-1\right)\right]^{1-\tanh \alpha} .
$$

To first order in $\epsilon, \delta r=r-r_{\Psi}$ satisfies the perturbed version of (10):

$$
\delta r^{\prime \prime}=-4 \pi \delta r\left(\Psi^{\prime}\right)^{2}-8 \pi \epsilon r_{\Psi} \Psi^{\prime} \phi^{\prime} .
$$

This linear differential equation for $\delta r$ is identical to (10) except for a inhomogeneous term. Its solution can therefore be expressed in terms of the two independent homogeneous solutions $r_{\Psi}$ and $\tau_{\Psi}$ as $[8]$

$$
\delta r=-\frac{4 \pi \epsilon}{M_{\Psi}} \int_{R}^{V} d W\left[r_{\Psi}(W) \tau_{\Psi}(V)-\tau_{\Psi}(W) r_{\Psi}(V)\right] r_{\Psi}(W) \Psi^{\prime}(W) \phi^{\prime}(W) .
$$

Note that this satisfies the proper boundary conditions $\delta r(R)=\delta r^{\prime}(R)=0$. By inserting this solution for $\delta r$ into (11), we obtain for the first variation of the Bondi mass

$$
M-M_{\Psi}=4 \pi \epsilon \int_{R}^{\infty} d V \tau_{\Psi} r_{\Psi} \Psi^{\prime} \phi^{\prime}
$$

But direct calculation gives

$$
\tau_{\Psi} r_{\Psi} \Psi^{\prime}=-\frac{R \sinh \alpha}{\sqrt{\pi}} e^{-2 \alpha \tanh \alpha}=\text { constant }
$$

so that (21) integrates to yield $M-M_{\Psi}=O\left(\epsilon^{2}\right)$.

Thus the static solutions are equilibrium configurations. For sufficiently small values of $\alpha$, the second variation of the Bondi mass will be positive, as in the flat space case. In that regime, small perturbations can be dissipated by mass loss due to scalar radiation. However, if there exists a perturbation which lowers the Bondi mass the static equilibria cannot be stable since further radiative mass loss would drive the system away from equilibrium. We next see that this indeed occurs in the strongly nonlinear regime. 


\section{B. The turning point instability}

The one-parameter family of static equilibria has kink potential $A_{\Psi}(\alpha)$, given by (15), which increases monotonically with $\alpha$ from $A_{\Psi}(0)=0$ until it reaches a maximum at the turning point $\alpha_{c} \approx 1.199$ satisfying $\alpha_{c} \tanh \alpha_{c}=1$. Above $\alpha_{c}, A_{\Psi}(\alpha)$ monotonically decreases to 0 as $\alpha \rightarrow \infty$. Thus, below $A_{\Psi}\left(\alpha_{c}\right)$, there are two static equilibria for each kink amplitude. Similarly, the mass $M_{\Psi}(\alpha)=0$ increases monotonically from $M_{\Psi}(0)=0$ to a maximum at the same turning point $\alpha_{c}$ and then decreases monotonically to the black hole limit, $M_{\Psi}(\alpha) \rightarrow R / 2$, as $\alpha \rightarrow \infty$.

The quantity $\kappa$, defined by $d M_{\Psi} / d \alpha=\kappa d A_{\Psi} / d \alpha$, plays an important role in the stability of this system. Explicitly,

$$
\kappa=4 \sqrt{\pi} R \sinh (\alpha) e^{-2 \alpha \tanh \alpha}
$$

Denoting $\dot{f}=d f / d \alpha$, it is easy to verify that $\dot{\kappa}_{\Psi} \approx .65 R>0$ for $\alpha=\alpha_{c}$.

These are precisely the criteria for the application of a theorem [2] regarding the onset of instability at a turning point in a one-parameter family of equilibria. We present here a finite dimensional version of the argument behind the theorem as applied to the present case. Proofs for both the finite dimensional and function space cases are given by Sorkin [2].

Consider then the configuration space $\left\{\Phi\left(A, X^{1}, \ldots, X^{n}\right)\right\}$ whose elements for each choice $\left(A, X^{i}\right)$ represent a function $\Phi\left(V ; A, X^{i}\right)$ with boundary conditions $\Phi\left(R ; A, X^{i}\right)=A$ and $\Phi\left(\infty ; A, X^{i}\right)=0$. Let $\Psi(\alpha)$ represent the one parameter family of static equilibria, regarding $\left(A_{\Psi}, X^{i}\right)$ as functions of $\alpha$. We now show that for some $\delta \alpha>0$ all the equilibria in the range $\alpha_{c}<\alpha<\alpha_{c}+\delta \alpha$ are unstable.

The proof is based upon the equilibrium conditions, $M_{\Psi, A}=\kappa$ and $M_{\Psi, i}=0$, where $f_{, A}=\partial_{A} f$ and $f_{, i}=\partial_{X^{i}} f$. Differentiating these conditions with respect to $\alpha$ along the equilibrium sequence gives

$$
\dot{\kappa}=M_{\Psi, A A} \dot{A}_{\Psi}+M_{\Psi, A i} \dot{X}^{i}
$$

and

$$
0=\dot{M}_{\Psi, i} \dot{X}^{i}=M_{\Psi, i A} \dot{A}_{\Psi} \dot{X}^{i}+\Delta
$$

where $\Delta=M_{\Psi, i j} \dot{X}^{i} \dot{X}^{j}$ represents the second variation of the mass with respect to the perturbation $\dot{X}^{i}$. Equation (25) implies that $\Delta=0$ at the turning point where $\dot{A}_{\Psi}=0$, , so that this mode has neutral stability. Taking a further derivative of (25) and evaluating at the turning point, we obtain

$$
\dot{\Delta}=-M_{\Psi, i A} \ddot{A}_{\Psi} \dot{X}^{i}=-\dot{\kappa} \ddot{A}_{\Psi} .
$$

As remarked above, $\dot{\kappa} \ddot{A}_{\Psi}>0$ at the turning point so that (26) implies the instability of this mode in a neighborhood $\alpha>\alpha_{c}$. 


\section{Unstable modes}

Since there are no other turning points, these general considerations suggest that the equilibria $\Psi$ are stable below $\alpha_{c}$ and unstable above $\alpha_{c}$, although the theorem only strictly implies the onset of instability in a neighborhood of $\alpha_{c}$. In order to provide further insight, we now consider specific behavior of the second variation of $M_{\Psi}$.

For an arbitrary perturbation, $\Phi=\Psi+\epsilon \phi$, we can follow the approach in Sec. II A which led to the expression (21) for the first variation of the mass and proceed further to determine the second variation. At order $\epsilon^{2}$, this leads to

$$
M-M_{\Psi}=2 \pi \epsilon^{2} \int_{R}^{\infty} d V \tau_{\Psi}\left(r_{\Psi} \phi^{\prime} \phi^{\prime}+2 \delta r \Psi^{\prime} \phi^{\prime}\right),
$$

where $\delta r$ is given by the integral (20). Because $\delta r$ depends nonlocally on the perturbation $\phi,(27)$ does not give straightforward information regarding the sign of the second variation. However, the first term in the integrand is positive definite and depends locally on the perturbation. Therefore, for perturbations confined to a sufficiently compact region, the first term dominates the $\delta r$ term and the second variation is positive. This leads us to consider large length scale perturbations in the search for unstable modes.

One such perturbation can be obtained from the static solution by combining a change in $\alpha$ with a translation,

$$
\delta \phi(V)=\Psi(V+\delta R ; \alpha+\delta \alpha)-\Psi(V ; \alpha)
$$

with $\delta \alpha$ and $\delta R$ adjusted to set $\delta \phi(R)=0$ so that the kink potential is held fixed. This requires

$$
\delta R=\frac{R\left(e^{-2(\alpha+\delta \alpha)} e^{4 \alpha \cosh (\alpha+\delta \alpha) / \cosh (\alpha)}-e^{2(\alpha+\delta \alpha)}\right)}{1-e^{4 \alpha \cosh (\alpha+\delta \alpha) / \cosh (\alpha)}} .
$$

For this perturbation, the change in $M$ can be worked out analytically to second order (using Maple) from (17), although the final expression is too lengthy to present here. The important point is that the second order variation of $M$ is positive for $\alpha<\alpha_{c}$, vanishes precisely for $\alpha=\alpha_{c}$ and turns negative in a neighborhood $\alpha>\alpha_{c}$. This corroborates our expectation that perturbations are stable below $\alpha_{c}$ and go unstable as we pass through the turning point. (It is curious that beyond the turning point $M-M_{\Psi}$ again becomes positive for values of $\alpha$ greater than approximately 2.0).

\section{GRAVITATIONAL COLLAPSE OF UNSTABLE KINKS}

We now examine the behavior of this system numerically, using a null cone evolution algorithm for nonlinear scalar waves developed in Ref's [9] and [4]. The algorithm is based upon the compactified radial coordinate $x=r /(R+r)$, so that $\mathcal{I}^{+}$is represented by a finite grid boundary, with $x=1 / 2$ at the mirror and $x=1$ at $\mathcal{I}^{+}$. The code has been tested to be globally second order accurate, i.e. the error in global quantities such as the Bondi mass is $O\left(\Delta x^{2}\right)$ in terms of the grid spacing $\Delta x$. 
In the case of a mirror boundary at zero potential (no kink), as studied in Ref. [3], a weak scalar field radiates completely to infinity, so that the final mass of the system is zero. However, above a critical strength, the scalar field undergoes gravitational collapse to form a horizon. In this case, some of the scalar energy is radiated to infinity and the remainder crosses the horizon and contributes to the final black hole mass. The mirror itself must fall into the horizon for otherwise it would continue to reflect the scalar field until all scalar energy were radiated to infinity. Near the critical strength, the sensitivity of the final mass is somewhat analogous to the critical behavior studied by Choptuik [10,11], except there is now a mass gap because the final black hole must have a mass larger than $R / 2$ in order to contain the mirror. In the present case of a mirror with nonvanishing kink potential, we would expect this behavior to be modified in several ways.

First, we consider kink potentials $A<A_{\Psi}\left(\alpha_{c}\right)$. In this case, two static equilibrium states $\Psi\left(\alpha_{-}\right)$and $\Psi\left(\alpha_{+}\right)$are possible, $\alpha_{-}<\alpha_{c}<\alpha_{+}$, with the former expected to be stable and the latter unstable. The double valued behavior of $M(A)$ is graphed in Figure 1. It shows that $M_{\Psi}\left(\alpha_{+}\right)>M_{\Psi}\left(\alpha_{-}\right)$. Consequently, the following scenarios are expected. A state close to $\Psi\left(\alpha_{-}\right)$should return to $\Psi\left(\alpha_{-}\right)$but a state close to $\Psi\left(\alpha_{+}\right)$could either evolve toward $\Psi\left(\alpha_{-}\right)$or collapse to a black hole. These scenarios have been confirmed by the following numerical simulations. (All these simulations are run setting $R=1$ ).

With kink potential $A=A_{\Psi}\left(\alpha_{-}\right) \approx 0.36563$ for $\alpha_{-}=1$, we study the stable equilibrium by evolving initial data of the form $\Phi=\Psi\left(\alpha_{-}\right)+\lambda(1-2 x) / 2 r$. For values of $\lambda$ less than a critical value $\lambda_{c} \approx 0.1929$, the system returns to the equilibrium state $\Psi\left(\alpha_{-}\right)$at late times. Fig. 2 graphs the corresponding time behavior of the Bondi mass showing its asymptotic approach to the equilibrium value $M \rightarrow M_{\Psi}\left(\alpha_{-}\right) \approx .60220$. For $\lambda>\lambda_{c}$, the system collapses to form a black hole.

Next, for the same kink potential $A \approx 0.36563$, we study the evolution of the unstable equilibrium by evolving initial data of the form $\Phi=\Psi\left(\alpha_{+}\right)+\lambda(1-2 x) / 2 r$. (Here $\alpha_{+} \approx$ 1.42429). Fig. 3 graphs the corresponding behavior of the Bondi mass. In this case, because of the instability of the static equilibrium $\Psi\left(\alpha_{+}\right)$, the system undergoes a dynamical change, even when $\lambda=0$ because of the perturbation introduced by discretization error in the initial data. For small $\lambda$, the state $\Psi\left(\alpha_{+}\right)$makes a transition to the stable state $\Psi\left(\alpha_{-}\right)$. But again there is a critical value $\lambda_{c} \approx(1.311) 10^{-4}$ above which the final state is a black hole.

Now consider a kink potential $A>A_{c}$, for which no static equilibria exist. With these boundary conditions, we would expect any initial state to undergo collapse to a black hole. We explore this by considering initial data of the form $\Phi=2\left(A_{c}+\lambda\right) /(r+R)$. Figs. 4 and 5 show the resulting behavior of $g$ as a function of $x$ at several times, for $\lambda=0.3$ and $\lambda=10^{-2}$, respectively. At late times, these states indeed form a black hole, as evidenced by the flattening of $g$, outside the cusp being formed at the black hole radius, as the exterior field sheds its "hair".

In summary, the behavior of this system is in complete accord with theoretical expectations. 


\section{ACKNOWLEDGMENTS}

We benefited from research support from the National Science Foundation under Grant PHY9510895 to the University of Pittsburgh and from computer time made available through the grant PHY850023P from the Pittsburgh Supercomputing Center. W.B. is grateful for the hospitality shown to him by the Relativity Group of the University of Pittsburgh, during his sabbatical year. He was supported in part by the Consejo de Investigación de la Universidad de Oriente, Venezuela. 


\section{REFERENCES}

[1] J. Ipser and G. Horwitz, Ap. J. 232, 863 (1979).

[2] R. Sorkin, Ap. J. 249, 254 (1981).

[3] R. Gómez, J. Winicour, and B. G. Schmidt, Phys. Rev. D 49, 2828 (1994).

[4] R. Gómez and J. Winicour, J. Math. Phys. 33, 1445 (1992).

[5] D. Christodoulou, Commun. Math. Phys. 105, 337 (1986).

[6] R. Gómez and J. Winicour, Phys. Rev. D 45, 2776 (1992).

[7] A. Janis, E. T. Newman, and J. Winicour, Phy. Rev. Letters 20, 878 (1968).

[8] E. A. Coddington and N. Levinson, Theory of ordinary differential equations (McGrawHill, New York, 1955), Chap. 9, p. 225.

[9] R. Gómez, J. Winicour, and R. Isaacson, J. Comp. Phys. 98, 11 (1992).

[10] M. Choptuik, in Approaches to Numerical Relativity, edited by R. d'Inverno (Cambridge University Press, Cambridge, 1992), p. 202.

[11] M. Choptuik, Phys. Rev. Lett 5, 9 (1993). 


\section{FIGURES}

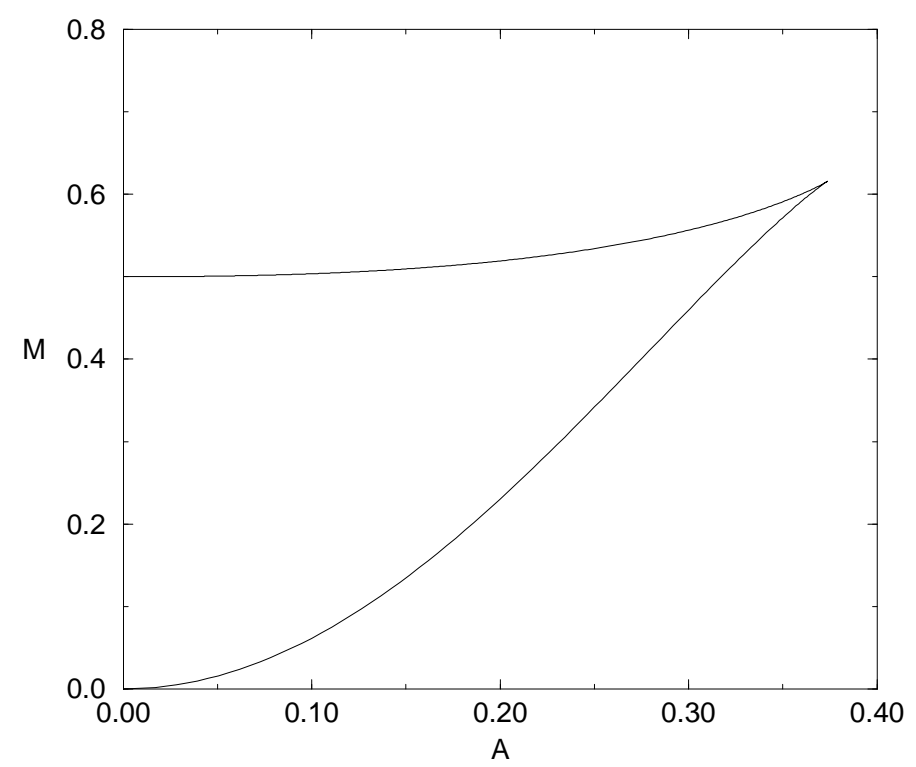

FIG. 1. Bondi mass as a function of amplitude, for the static equilibria.

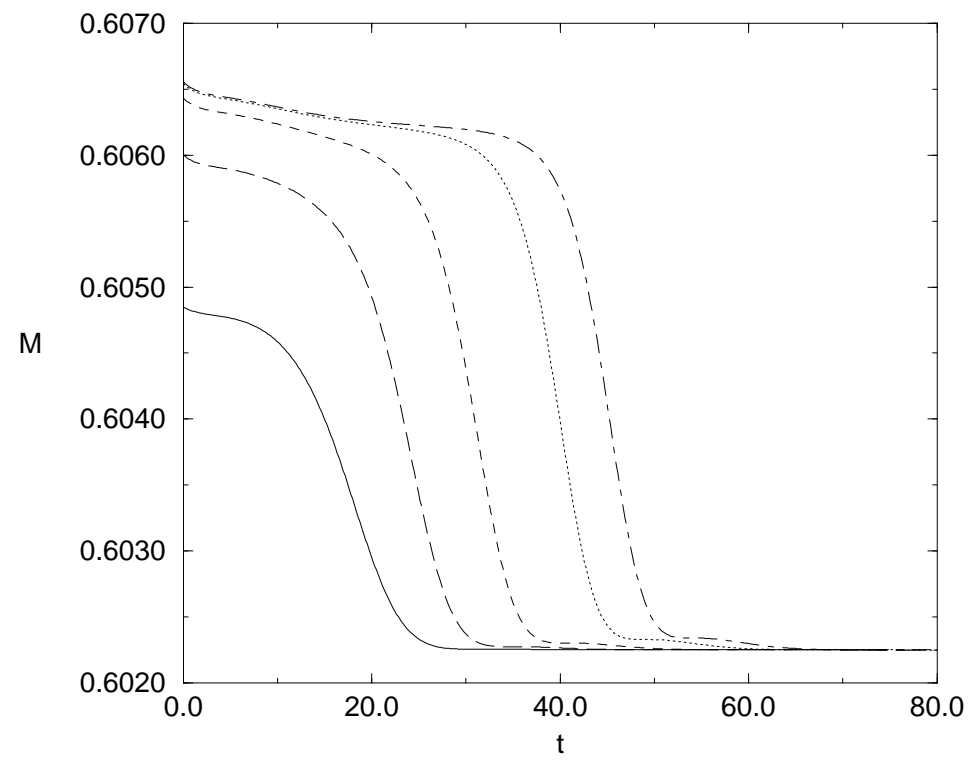

FIG. 2. Bondi mass as a function of Bondi time for perturbations of a stable equilibrium. Different curves represent the different values of the perturbation parameter $\lambda$ : 0.15 (continuous line); 0.18 (dashed line); 0.19 (small-dashed line); 0.1925 (dotted line); 0.1928 (dot-dashed line). The system collapses to a black hole for $\lambda$ greater than $\lambda_{c} \approx 0.1929$. 


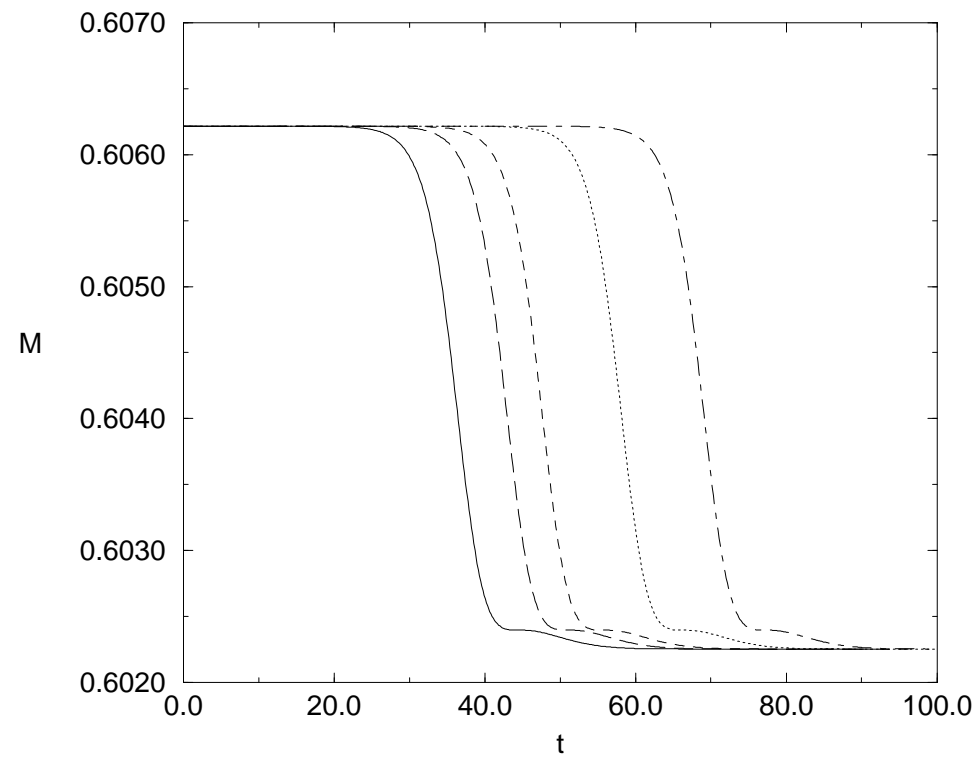

FIG. 3. Bondi mass as a function of Bondi time for perturbations of an unstable equilibrium. The different values of the perturbation parameter $\lambda$ are: 0 (continuous line); $10^{-4}$ (dashed line); (1.2) $10^{-4}$ (small-dashed line); (1.3) $10^{-4}$ (dotted line); (1.31) $10^{-4}$ (dot-dashed line). For these values the system makes a transition to the stable equilibrium. The system collapses to a black hole for $\lambda_{c} \approx(1.311) 10^{-4}$.

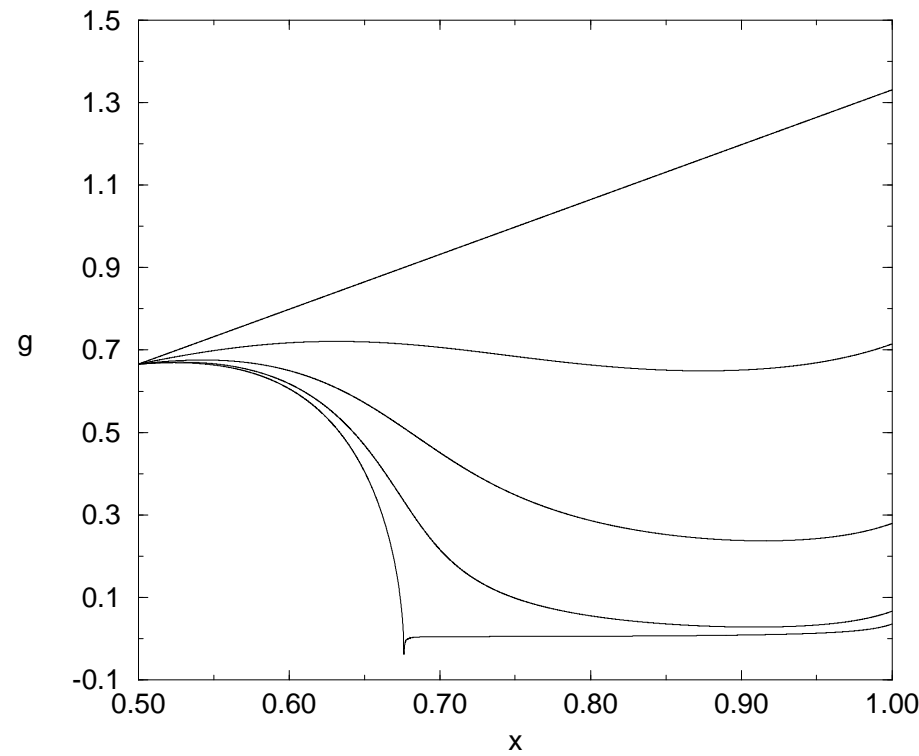

FIG. 4. Evolution of $g$. The initial data is $g(0, x)=2 x\left(A_{c}+\lambda\right)$, for $\lambda=0.3$. The upper line represents $u=0$; and the lower one, $u=1.19$. The system collapses to a black hole. 


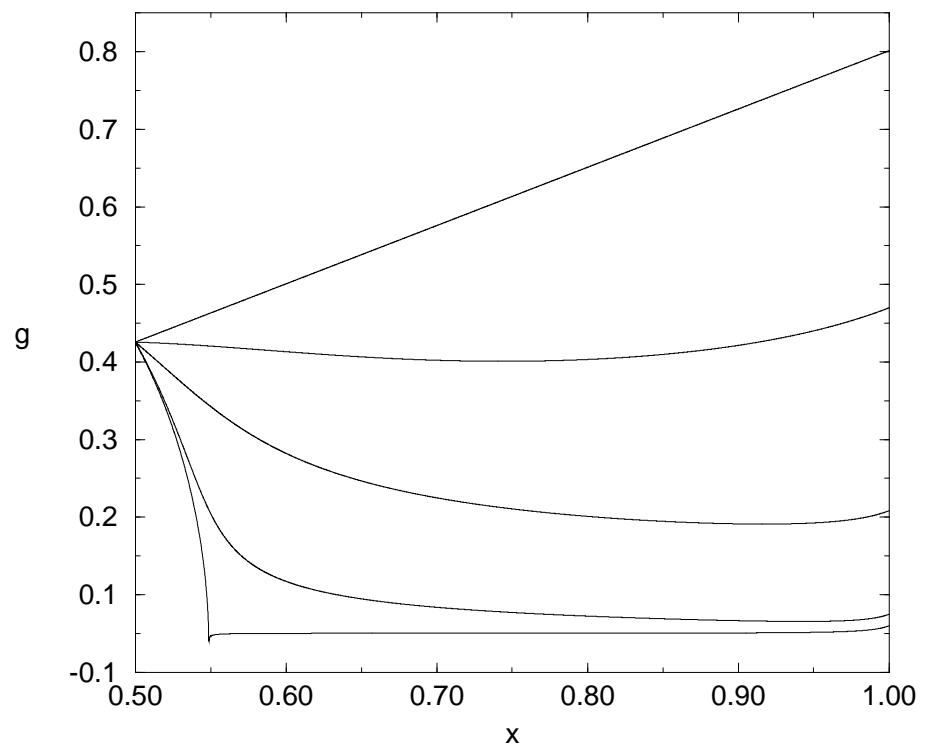

FIG. 5. Evolution of $g$. The initial data is $g(0, x)=2 x\left(A_{c}+\lambda\right)$, for $\lambda=10^{-2}$. The upper line represents $u=0$, and the lower one $u=15.33$. The system collapses to a black hole. 